\title{
Hydrocortisone, ascorbic acid, and thiamine (HAT) for sepsis and septic shock: a meta-analysis with sequential trial analysis
}

\author{
Weilan $\mathrm{Na}^{1}$, Huili Shen ${ }^{2}$, Yichu $\mathrm{Li}^{1}$ and Dong Qu ${ }^{1 *}$
}

\begin{abstract}
Background: Sepsis is a primary global health threat and costs a lot, requiring effective and affordable treatments. We performed this meta-analysis to explore the treatment of hydrocortisone, ascorbic acid, and thiamine (HAT) in sepsis and septic shock.

Methods: We searched Ovid MEDLINE, Embase, and the Cochrane Central Register of Controlled Trials from inception to August 14, 2021. We included randomized controlled trials (RCTs) that evaluated the HAT treatments in sepsis and septic shock. The primary outcome was the change in SOFA score over the $72 \mathrm{~h}$. The second outcomes were the hospital, and 28-/30-day mortality, the duration of vasopressors, PCT clearance, hospital length of stay (LOS), and ICU LOS. We performed a subgroup analysis and a trial sequential analysis (TSA). The Der Simonian-Laird random-effects models were used to report the pooled risk ratios (RR) or mean difference (MD) with confidence intervals (CI).

Results: Nine RCTs, enrolling 1427 patients of sepsis and septic shock treated with HAT (717) or only standard care (710), were included. There was a significant difference between the two groups in the change in SOFA score over the first $72 \mathrm{~h}(\mathrm{MD} 0.65,95 \% \mathrm{Cl} 0.30$ to 1.00), the duration of vasopressors (MD $-18.16,95 \% \mathrm{Cl}-25.65$ to -10.68$)$ and the PCT clearance (MD 14.54, 95\% Cl 0.64 to 28.43). In addition, there was no significant difference in the hospital mortality (RR $1.07,95 \% \mathrm{Cl} 0.85$ to 1.34 ), the $28-/ 30$-day mortality (RR $0.96,95 \% \mathrm{Cl} 0.80$ to 1.15 ), the hospital LOS (MD $0.78,95 \%$ $\mathrm{Cl}-0.30$ to 1.86 ), and ICU LOS (MD $0.12,95 \% \mathrm{Cl}-0.53$ to 0.78$)$.
\end{abstract}

Conclusions: The HAT combination improves the SOFA score in the first $72 \mathrm{~h}$ and reduces the duration of vasopressors in patients with sepsis. Given the minor mean difference of the change in SOFA score, the mortality benefit has not been observed.

Trial registration: PROSPERO, CRD42020203166.

Keywords: Sepsis, Meta-analysis, Hydrocortisone, Ascorbic acid, Thiamine

\section{Introduction}

Sepsis is a life-threatening organ dysfunction syndrome due to a dysregulated host response to infection [1]. It has been recognized as a primary health threat with high

\footnotetext{
*Correspondence: qudong2012@126.com

1 Department of Critical Medicine, Children's Hospital Affiliated

to the Capital Institute of Pediatrics, NO.2 Ya Bao Road, Chaoyang District, Beijing 100020, China

Full list of author information is available at the end of the article
}

morbidity and mortality, contributing to up to 5.3 million deaths worldwide each year and cost a lot [2]. Given the tremendous financial burden of sepsis, more effective but affordable treatments were required. A retrospective study, conducted by Marik et al. [3], first found that the combination of hydrocortisone, ascorbic acid, and thiamine (HAT) effectively reduced mortality and prevented organ dysfunction for sepsis and septic shock patients. original author(s) and the source, provide a link to the Creative Commons licence, and indicate if changes were made. The images or other third party material in this article are included in the article's Creative Commons licence, unless indicated otherwise in a credit line to the material. If material is not included in the article's Creative Commons licence and your intended use is not permitted by statutory regulation or exceeds the permitted use, you will need to obtain permission directly from the copyright holder. To view a copy of this licence, visit http://creativecommons.org/licenses/by/4.0/. The Creative Commons Public Domain Dedication waiver (http://creativecommons.org/publicdomain/zero/1.0/) applies to the data made available in this article, unless otherwise stated in a credit line to the data. 
Hydrocortisone is considered as a typical adjuvant therapy for septic shock, based on the reversal of relative adrenal insufficiency. Low-dose hydrocortisone treatment may rapidly induce hemodynamic stabilization by reducing nitric oxide formation and can regulate the complex immune network in a widely ranging way [4]. Ascorbic acid, well known as Vitamin C, is an important antioxidant and an essential cofactor for biosynthesis and cell metabolization. In patients with sepsis and septic shock, there is a prevalent vitamin $\mathrm{C}$ deficiency trend upon admission to intensive care, resulting from increased oxidative stress [5]. Thiamine is referred to vitamin $\mathrm{B} 1$, an essential intermediate affecting pyruvate flux to the Krebs cycle. Thiamine deficiency has also been described in septic patients and led to increase lactate production via aerobic metabolism changes [6]. Polypharmacy act synergistically in multiple overlapping ways. This combination's biologic basis is the protective synergistic effect of hydrocortisone and vitamin $\mathrm{C}$ that ascorbic acid can restore glucocorticoid receptor function negatively affected by oxide [7]. Septic shock is associated with endothelial barrier dysfunction, which can be synergistically attenuated by hydrocortisone and vitamin $\mathrm{C}$ via the reversal of $\mathrm{p} 53$ and phosphorylated cofilin downregulation [8]. They also increase tight junctions between endothelial and epithelial cells, which preserves endothelial function and microcirculatory flow. Better yet, both are necessary for the synthesis of catecholamines and increase the sensitivity of vascular vasopressors [9]. In addition, thiamine, with glucocorticoids and vitamin $\mathrm{C}$, can attenuate mitochondrial damage and promote mitochondrial function, which synergistically benefits a lot [10].

The HAT combination is simple, affordable, and theoretically beneficial for septic patients. However, as several RCTs showed conflicting results, the HAT therapy did not appear to reduce the mortality and was not supported for routine use [11-13]. A large retrospective cohort study of US adults with septic shock revealed that the use of HAT therapy increased significantly after Marik et al. [3] proposed the HAT combination, with more than $40 \%$ of the study hospitals using it [14]. This early adoption was due to high media attention rather than robust evidence of efficacy, which may carry unintentional risks. When considered in conjunction with recent studies, the combination seems to be a promising treatment, and this meta-analysis aimed to evaluate the effects of hydrocortisone, ascorbic acid, and thiamine given together in sepsis and septic shock.

\section{Methods}

\section{Data sources and search strategies}

The systematic review was performed following the Cochrane Handbook guidelines for Systematic Reviews of Interventions and the PRISMA statement [15, 16]. Trial sequential analysis (TSA) was used to increase the reliability of the meta-analysis and estimate the required information size [17]. The protocol was preregistered on PROSPERO, ID: CRD42020203166. Ovid MEDLINE, Embase, and the Cochrane Central Register of Controlled Trials (CENTRAL) were searched using the search strategies (Appendix 1) on August 14, 2021. In addition, the reference lists of the included studies and relevant meta-analyses were checked.

\section{Study selection}

Inclusion criteria were as fellow: patients ( $>18$ years) with sepsis or septic shock; patients receiving HAT treatments in the intervention group; randomized controlled trials. As the HAT combination was first proposed in 2016, the definition of sepsis-3 was accepted. Considering the common use of glucocorticoids in sepsis and septic shock, we did not exclude the use in the control group and all types of glucocorticoids were included. There was no language restriction.

According to the inclusion criteria, two authors independently screened the titles and abstracts and then did full-text reviews of selected studies. Disagreements were resolved by consultation with a third member of the review team.

\section{Data extraction}

Two authors extracted data independently and consensus was reached. The data extracted included the following: authors, publication year, country, study design, number, inclusion and exclusion criteria, demographics, outcome measures and study results, independently.

\section{Study endpoints}

The primary outcome was the change in Sequential Organ Failure Assessment (SOFA) score over 72 h. Secondary outcomes were as follows: the hospital mortality, 28-/30-day mortality, the duration of vasopressors, procalcitonin (PCT) clearance, hospital length of stay (LOS), and ICU LOS.

\section{Subgroup analysis and sensitivity analysis}

For the subgroup analysis, septic shock was assessed as a subgroup. A sensitivity analysis was performed for 


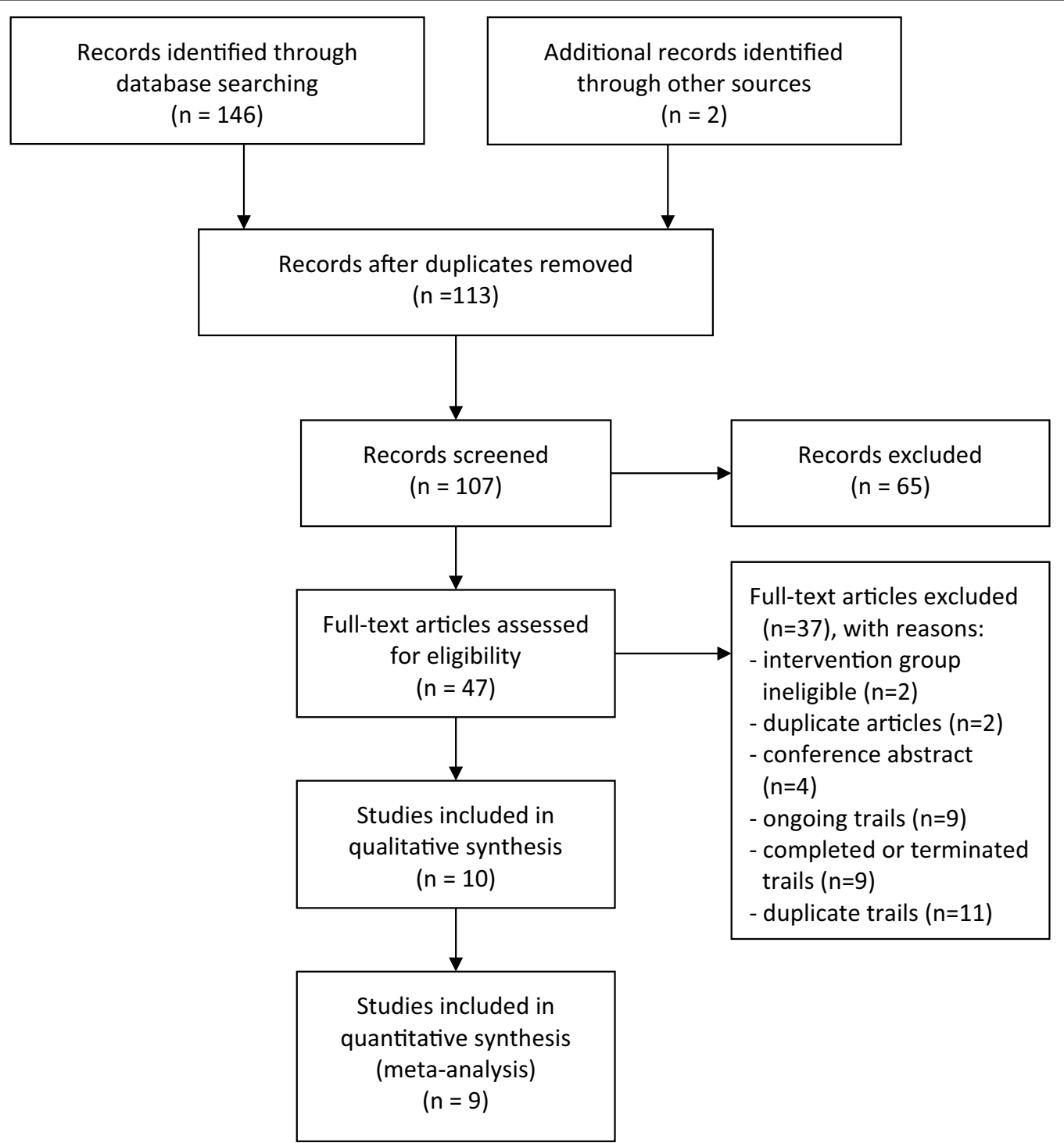

Fig. 1 Flow diagram

trials that excluded patients with renal failure at enrollment. For those analyses, the outcome was the change of SOFA score over $72 \mathrm{~h}$.

\section{Assessment risk of bias}

The Cochrane Risk-of-Bias Tool was used to assess the risk of bias in the domains of selection, performance, detection, attrition, and reporting. Two authors completed the assessment independently, and disagreements were resolved by consensus or the third author.

\section{Statistical analysis}

Data were analyzed using Review Manager 5.3 and TSA 0.9.5.10 Beta program. We presented results as forest plots through the risk ratios (RRs) with 95\% confidence intervals (CI) for dichotomous data. Forest plots using the mean difference (MD) with 95\% CI were performed for continuous data. The heterogeneity was defined via $I^{2}$ statistic. An $I^{2}$-value $>50 \%$ was considered heterogeneity. Random-effects model was used for all pooled analysis. If the value of $P$ was less than 0.05 , regarded as statistically significant. We also conducted a TSA to control random errors and calculate the required information size (RIS) based on a two-sided $a$ of $0.05, \beta$ of $80 \%$. 


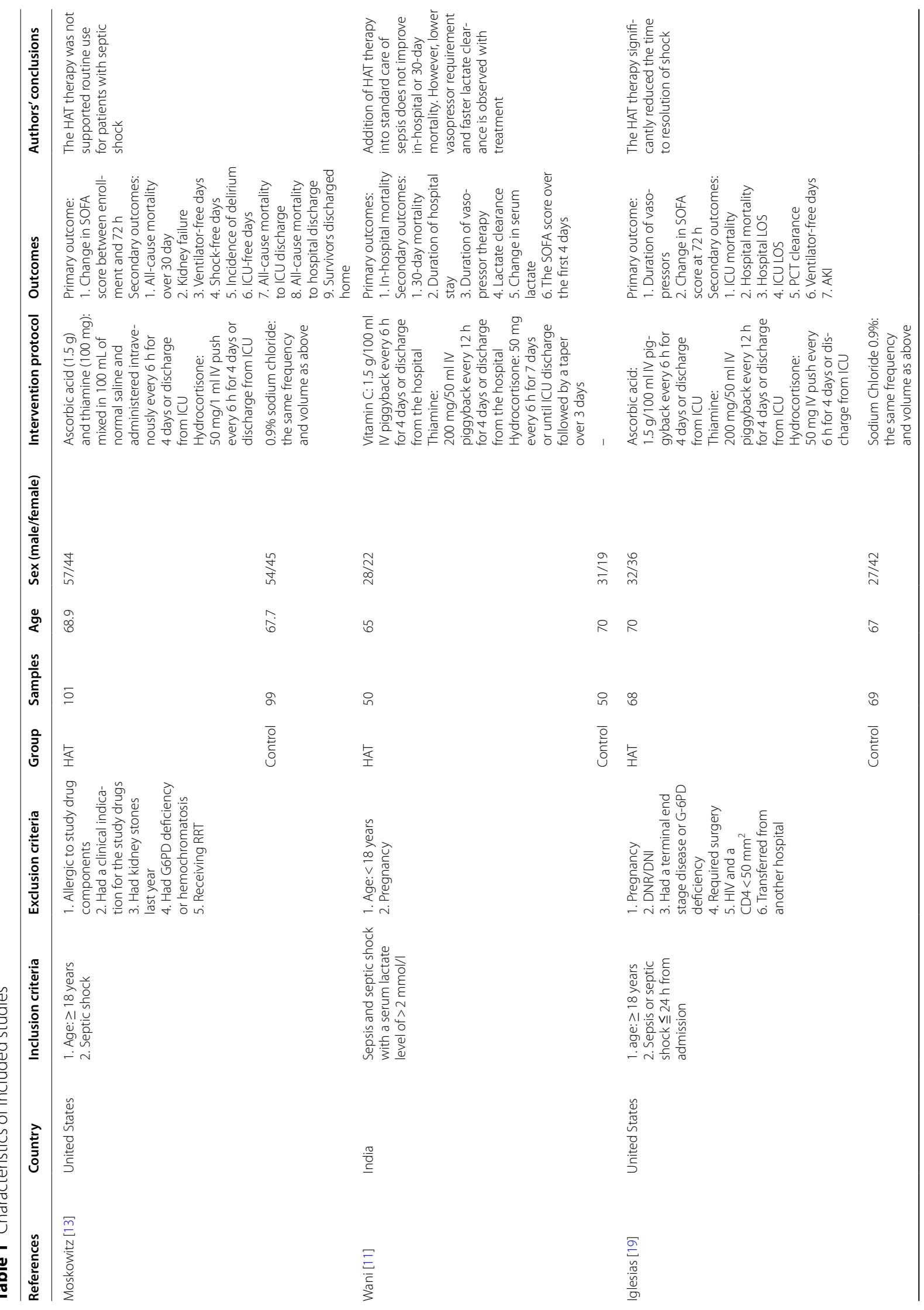




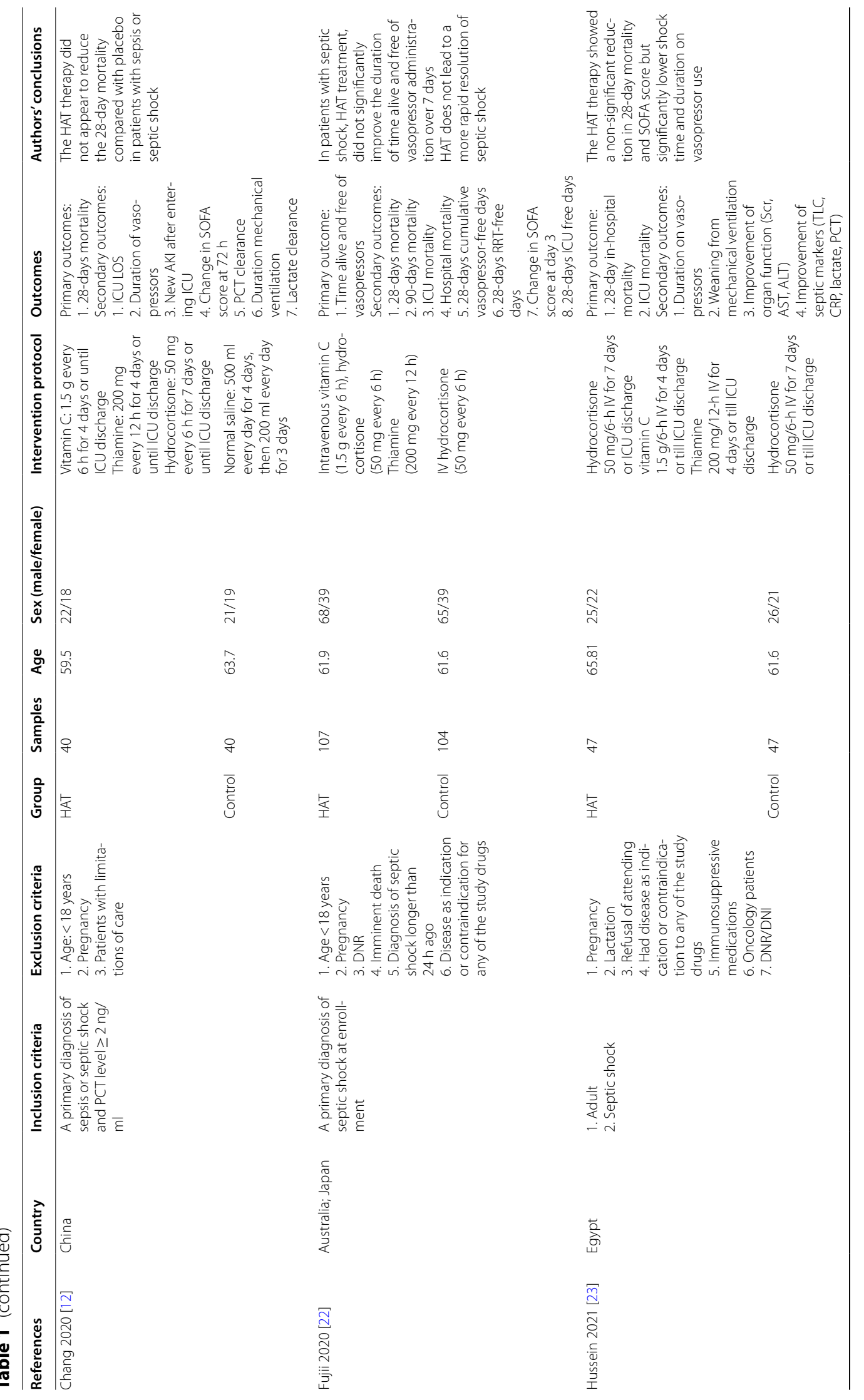




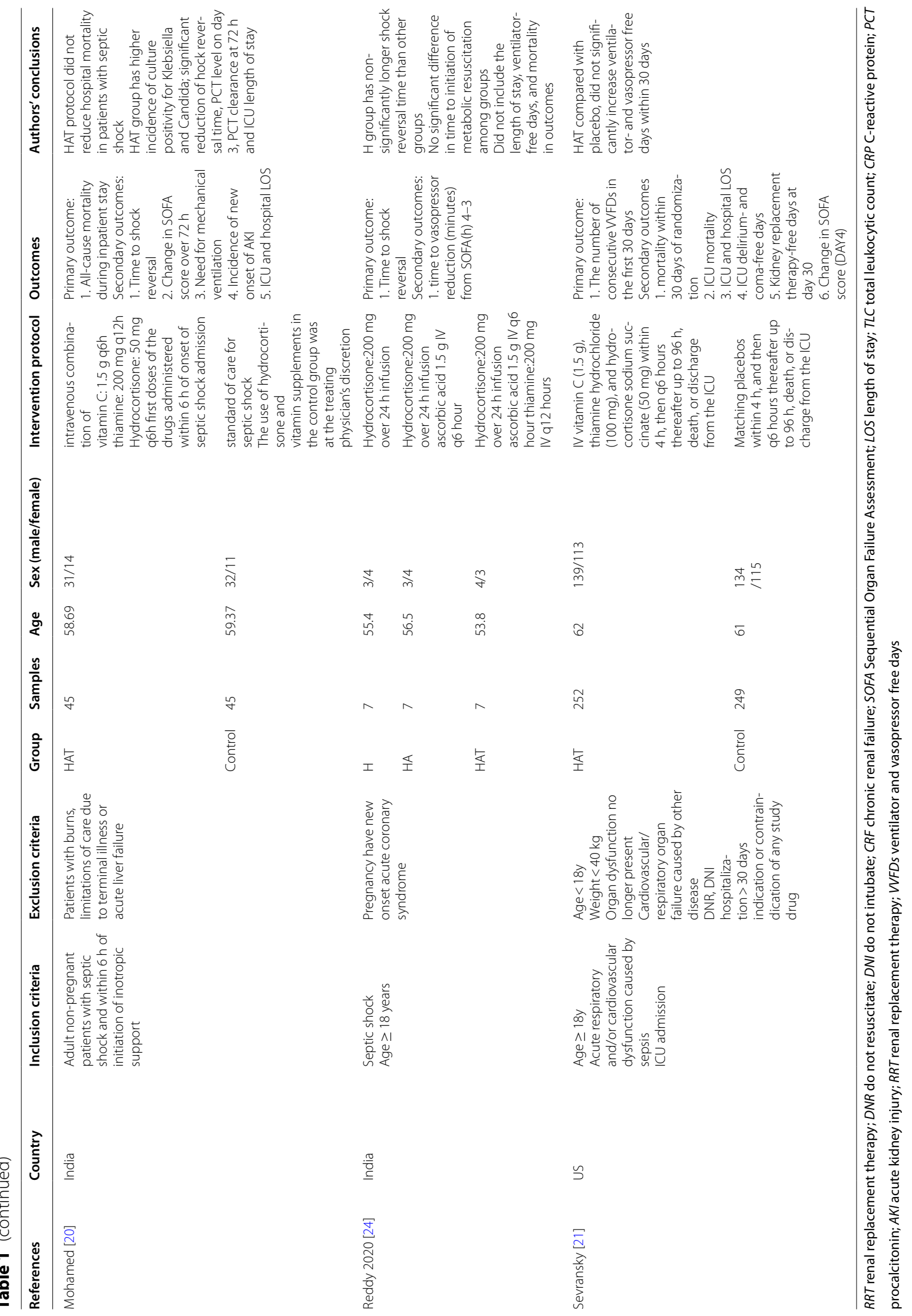




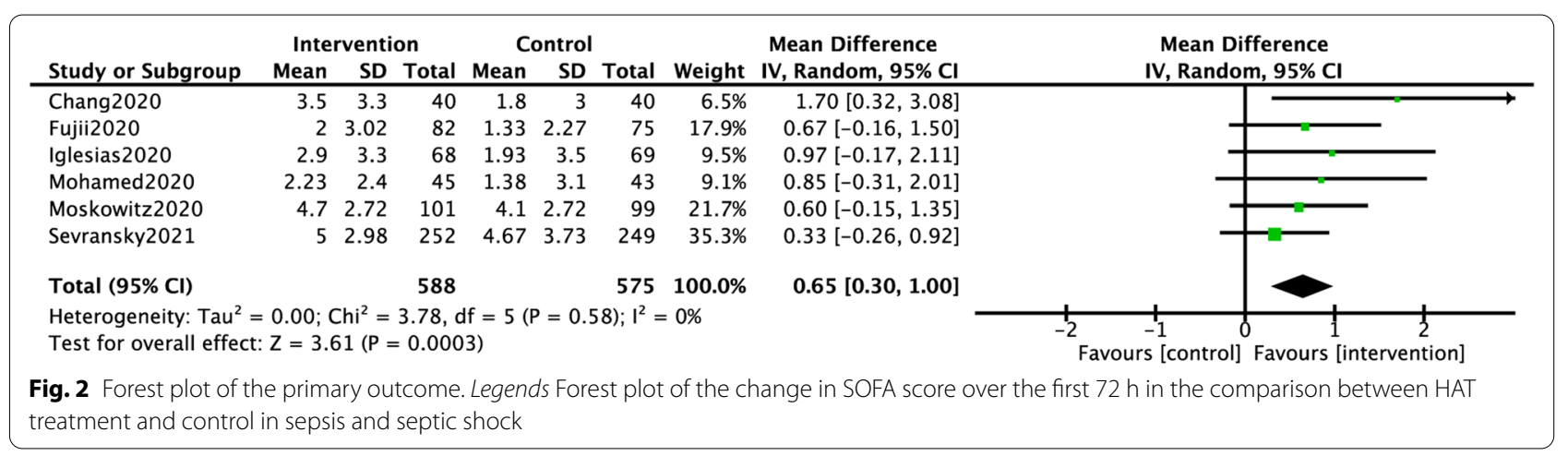

\section{Results}

\section{Search results and study characteristics}

The search retrieved 148 results up to August 14, 2021. After the elimination of duplicates, 113 studies were eligible based on the assessment of the title and abstract.

Then 47 trials were reviewed with the full text; 10 were included in the systematic review and 9 were included in the meta-analysis finally (Fig. 1). The excluded study did not contain the predefined outcomes [18]. One thousand four hundred and twenty seven patients with sepsis and septic shock were included in the meta-analysis (717 in the HAT treatment group and 710 in the control group). The characteristics of each trial were summarized in Table 1. The included studies differed in the application of glucocorticoids. In three studies [11-13], patients in the control group were only treated with the standard care for sepsis and septic shock, including broad-spectrum antibiotics, intravenous fluids, vasopressors, and mechanical ventilation. In another three studies [19-21], intensivists were allowed to order open-label corticosteroid therapy as deemed necessary. In three other studies [22-24], patients in the control group were routinely given low doses of glucocorticoids. In addition, the severity of sepsis was varied. Four trials focused on patients with sepsis including those with septic shock [11, 12, 19, 21 and the others only focused on patients with septic shock [13, 20, 22-24].

\section{Outcomes}

The forest plot of the primary outcomes was shown in Fig. 2. The change in SOFA score over $72 \mathrm{~h}$ was reported in six studies (588 in the HAT group and 575 in the control group). A significant reduction in SOFA score was revealed, with the use of HAT, and there was no significant heterogeneity (MD 0.65, 95\% CI 0.30 to 1.00 , $\left.P=0.0003 ; I^{2}=0 \%, P_{H}=0.58\right)$.

For the secondary outcomes, the pooled RR of hospital mortality and 28-/30-day mortality did not reach the statistical significance (RR $1.07,95 \%$ CI 0.85 to 1.34 and RR $0.96,95 \%$ CI 0.80 to 1.15 , respectively) (Fig. 3a and $\mathrm{b})$. The pooled results of the duration of vasopressors revealed a significant reduction in the HAT treatment group, with no heterogeneity (MD $-18.16,95 \% \mathrm{CI}$ -25.65 to $-10.68, P<0.01 ; I^{2}=29 \%, P_{H}=0.65$; Fig. $3 \mathrm{c}$ ). For the PCT clearance, there was statistical significance between two groups (MD 14.54, 95\% CI 0.64 to 28.43; Fig. 3d). In addition, there were no significant differences in the hospital and ICU LOS between the two groups with pooled MD of 0.78 (95\% CI -0.30 to 1.86$)$ and 0.12 (95\% CI -0.53 to 0.78 ), respectively (Fig. 3e and f).

For the subgroup analysis of septic shock, the result was presented in Fig. 4a. Four in seven studies were included and the HAT treatment showed a significant improvement in the SOFA score over $72 \mathrm{~h}$ (MD 0.67, 95\% CI 0.17 to 1.18 ). For the sensitivity analysis, only two trials excluded the patients with renal failure at enrollment and there was also statistically significant (MD 1.03, 95\% CI 0.07 to 1.99 ; Fig. 4b).

\section{Trial sequential analysis results}

TSA showed the adjusted pooled effect of the change in SOFA score over $72 \mathrm{~h}$ was 0.56 (95\% CI 0.23 to 0.89 ) (Fig. 4c). The red cumulative $z$ curve crossed the blue trial sequential boundary and the conventional boundary, indicating that the result was stable and statistically significant. In addition, the RIS of 1038 patients had been accrued, which indicated a sufficient number of studies.

\section{Publication bias and risk of bias}

The presence of publication bias for the primary outcome was tested and the funnel plot did not show the existence of publication bias via a visual inspection (Fig. 5a). For the risk of bias, the lack of blinding led to the performance bias and detection bias rated the highest (high risk of biases in 5/10 trials) (Fig. 5b). 


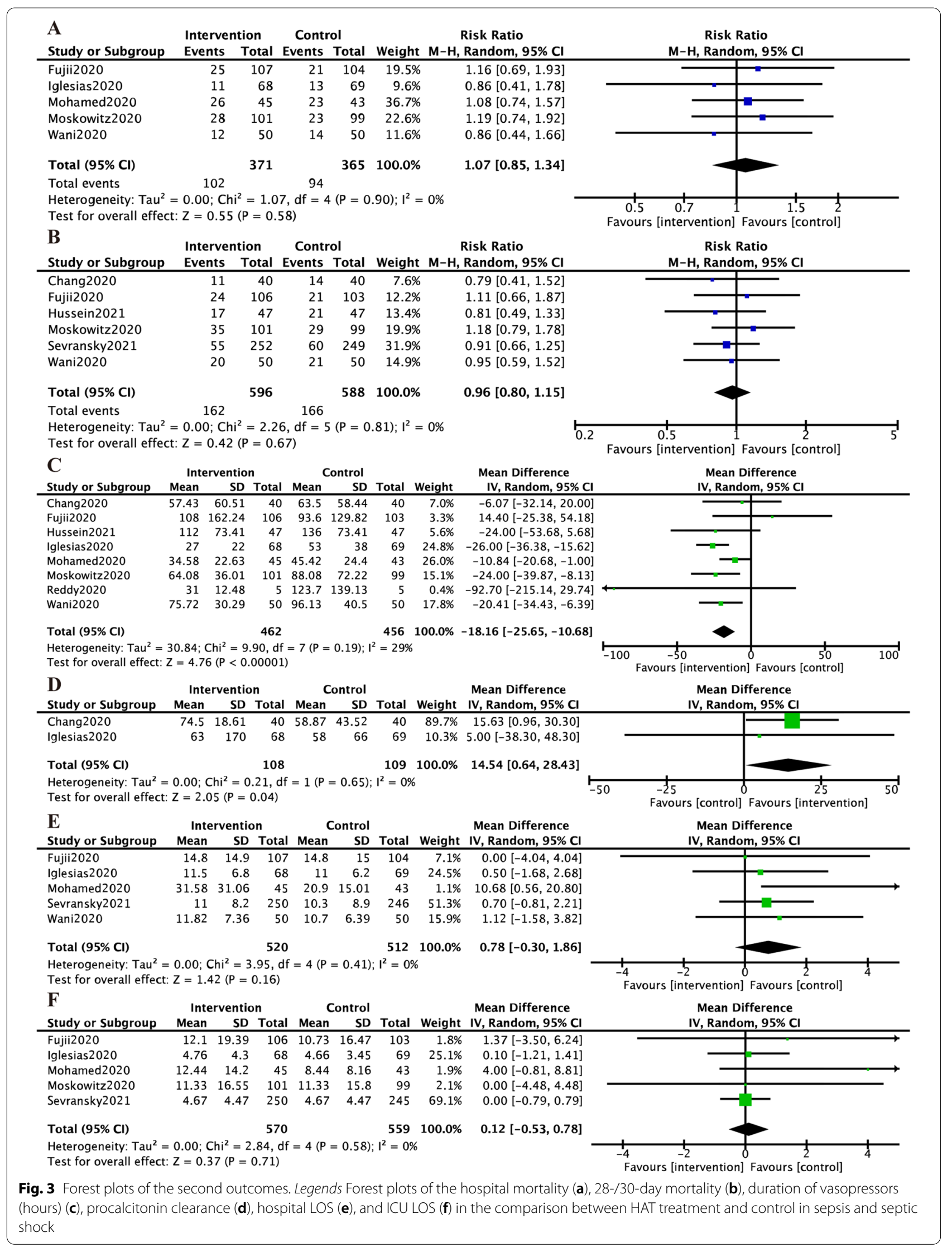




\section{Discussion}

In this systematic review, the combination of hydrocortisone, ascorbic acid, and thiamine led to the reduction of SOFA score over $72 \mathrm{~h}$, the duration of vasopressors and the improvement of PCT clearance. However, the HAT combination did not show benefit in the mortality, the duration of mechanical ventilation, and hospital or ICU LOS.

According to the Sepsis-3 clinical criteria, the diagnosis of sepsis has emphasized organ dysfunction which was represented by the increase of two points or more SOFA score. In addition, a change in the SOFA score was accepted by the European Medicines Agency as a surrogate marker of efficacy in exploratory trials of novel therapeutic agents in sepsis [25]. Moreover, organ dysfunction was associated with about $10 \%$ increase in mortality [26]. Therefore, we selected the change of SOFA score over the $72 \mathrm{~h}$, rather than hospital mortality which was chosen in the PROSPERO registry, as the primary outcome. In this meta-analysis, there was a significant effect of the intervention on the change of SOFA score, but this did not be translated into a mortality benefit. First, hospital mortality is all-cause mortality and is influenced by many factors, such as comorbidities. Most included studies did not exclude the patients with a terminal end-stage disease or with imminent death, which may underestimate the therapeutic effect. In addition, the pooled effect (MD $0.65,95 \%$ CI 0.30 to 1.00 ) was so minor and did not achieve the minimal clinically important difference, which was set as a 2 -point difference [1, 13]. However, caution should be paid to the interpretation of the statistical results. It is valid only if the SOFA change is clinically relevant. The SOFA score focused on the early recovery of organ function and was assessed only if the patients remained in the ICU on the third day. Considering of the potential endpoints, such as death or recovery leading to early discharge from ICU, which increased the bias of competing risk [22]. Although the pooled effect of both meta-analysis and TSA supported the reliability and stability, the mean difference was small and we should still be cautious to evaluate the effect of HAT combination on the organ function.

Numerous retrospective studies showed a conflicting result on the mortality benefits, but the HAT combination did not provide significant survival benefits in this meta-analysis. In the study of Wald et al. [27], the HAT combination was found to be associated with lower mortality in pediatric septic shock, and the improvement seemed to be primarily associated with reduced early deaths. In addition, Marik et al. [3] found that the early use of the HAT combination appeared to significantly affect patients' hospital mortality with sepsis and septic shock. According to clinical pharmacologic knowledge and pathophysiological mechanisms, we speculated that the early use of the HAT combination may make sense for patients at different sepsis stages. Noteworthy, the HYVCTTSSS trial, conducted by Chang et al. [12], showed that the HAT group got a better therapeutic effect than the control group in the subgroup, where patients were diagnosed with sepsis within $48 \mathrm{~h}$, reflected mainly in the improvement of mortality. Hence, there is reason to believe that early treatment can lead to higher survival rates.

Theoretically, glucocorticoids and vitamin $\mathrm{C}$ have the ability to synergistically increase the sensitivity of vasopressors, which was also reflected in the results of this meta-analysis. In the HAT group, the duration of vasopressors was significantly reduced. Early liberation from vasopressor therapy means early recovery from septic shock. Although it could provide a more stable hemodynamic basis for subsequent treatment, the infectious source control remains the key for the mortality. The prognostic value of serum PCT in septic patients has been widely investigated and PCT non-clearance are strongly associated with all-cause mortality [28, 29]. Although there was statistically significant, caution should be exercised when interpreting the unstable results. In the ViCTOR Trial [20], the hospital LOS was significantly higher in the HAT group. However, after adjusting for outliers, the average LOS between the study groups did not significantly differ, which was consistent with the pooled effect of this meta-analysis.

Two meta-analyses regarding the effects of HAT therapy were published recently. Both Zayed et al. [30] and Somagutta et al. [31] concluded that HAT therapy significantly improved the SOFA score but appeared not to have significant benefits in the mortality, which was consistent with the results of this meta-analysis with a larger sample size. However, in our meta-analysis, instead of focusing on mortality, we set the change in SOFA score

\footnotetext{
(See figure on next page.)

Fig. 4 Subgroup analysis, sensitivity analysis and TSA of the primary outcome. Legends: a Forest plots of the subgroup analysis for the change in SOFA score over the first $72 \mathrm{~h}$. Septic shock was assessed as a subgroup. b Forest plots of the sensitivity analysis for the change in SOFA score over the first $72 \mathrm{~h}$. Trials that excluded patients with renal failure at enrollment was assessed. c Trial sequential analysis for the change in SOFA score over the first $72 \mathrm{~h}$. The blue cumulative $\mathrm{z}$ curve crossed the conventional monitoring boundary and the red trial sequential boundary for benefit (the pooled effect, $0.56 ; 95 \% \mathrm{Cl} 0.23-0.89 ; I^{2}=0 \%$ ). The required information size (RIS) was 1038 (a two-sided a of $0.05, \beta$ of $80 \%$ )
} 


\section{A} Intervention
Mean SD Total

Control

Mean Difference

Mean Difference

Study or Subgroup Mean SD Total Mean SD Total Weight IV, Random, $95 \% \mathrm{Cl}$

3.1.1 sepsis and septic shock

$\begin{array}{lllllll}3.5 & 3.3 & 40 & 1.8 & 3 & 40 & 6.5 \%\end{array}$

Iglesias 2020

2.93 .3

$\begin{array}{lll}1.8 & 69 & 9.5 \%\end{array}$

Sevransky2021
Subtotal $(95 \% \mathrm{Cl})$

$\begin{array}{lllllll}5 & 2.98 & 252 & 4.67 & 3.73 & 249 & 35.3 \%\end{array}$

$1.70[0.32,3.08]$

$0.97[-0.17,2.11]$

360

$358 \quad 51.3 \%$

$0.33[-0.26,0.92]$

Heterogeneity: $\mathrm{Tau}^{2}=0.21 ; \mathrm{Chi}^{2}=3.63, \mathrm{df}=2(\mathrm{P}=0.16) ; \mathrm{I}^{2}=45 \%$

Test for overall effect: $Z=2.05(P=0.04)$

3.1.2 septic shock

Fujili2020

Moskowitz2020

$\begin{array}{rrrrr}2 & 3.02 & 82 & 1.33 & 2.27 \\ 2.23 & 2.4 & 45 & 1.38 & 3.1\end{array}$

$\begin{array}{ll}75 & 17.9 \% \\ 43 & 9.1 \%\end{array}$

$4.7 \quad 2.72$

$\begin{array}{lrrrr}101 & 4.1 & 2.72 & 99 & 21.7 \%\end{array}$

$\begin{array}{rr}99 & 21.7 \% \\ \mathbf{2 1 7} & \mathbf{4 8 . 7 \%}\end{array}$

Muskowitz2020

228

Heterogeneity: $\mathrm{Tau}^{2}=0.00 ; \mathrm{Chi}^{2}=0.13, \mathrm{df}=2(\mathrm{P}=0.94) ; \mathrm{I}^{2}=0 \%$

Test for overall effect: $Z=2.62(P=0.009)$

Total (95\% Cl)

588

$575 \quad \mathbf{1 0 0 . 0 \%}$

Heterogeneity: $\mathrm{Tau}^{2}=0.00 ; \mathrm{Chi}^{2}=3.78, \mathrm{df}=5(\mathrm{P}=0.58) ; \mathrm{I}^{2}=0 \%$

Test for overall effect: $Z=3.61(P=0.0003)$

Test for subgroup differences: $\mathrm{Chi}^{2}=0.08, \mathrm{df}=1(\mathrm{P}=0.78), \mathrm{I}^{2}=0 \%$

$0.67[0.17,1.18]$

B

Intervention Control Mean Difference

\begin{tabular}{|c|c|c|c|c|c|c|c|c|}
\hline Cha & 3.5 & 3.3 & 40 & 1.8 & 3 & 40 & $35.0 \%$ & $2,3.08]$ \\
\hline Fujii & 2 & 3.02 & 82 & 1.33 & 2.27 & 75 & $65.0 \%$ & $0.67[-0.16,1.50]$ \\
\hline
\end{tabular}

Total $(95 \% \mathrm{Cl})$

122

$115 \quad 100.0 \%$

Heterogeneity: $\mathrm{Tau}^{2}=0.19 ; \mathrm{Chi}^{2}=1.57, \mathrm{df}=1(\mathrm{P}=0.21) ; \mathrm{I}^{2}=36 \%$

Test for overall effect: $Z=2.10(P=0.04)$

$1.03[0.07,1.99]$

C

RIS is a Two-sided graph

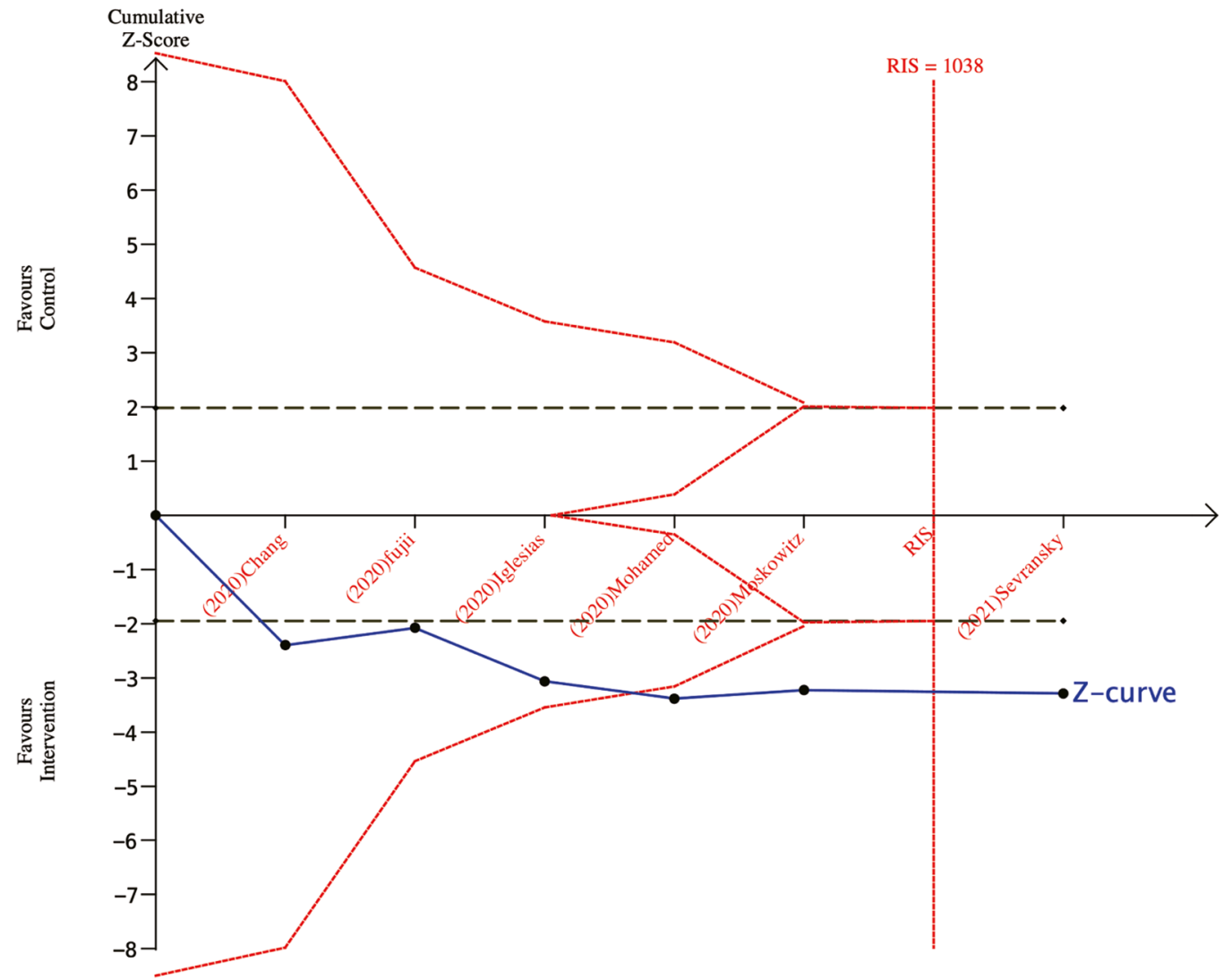

Fig. 4 (See legend on previous page.) 


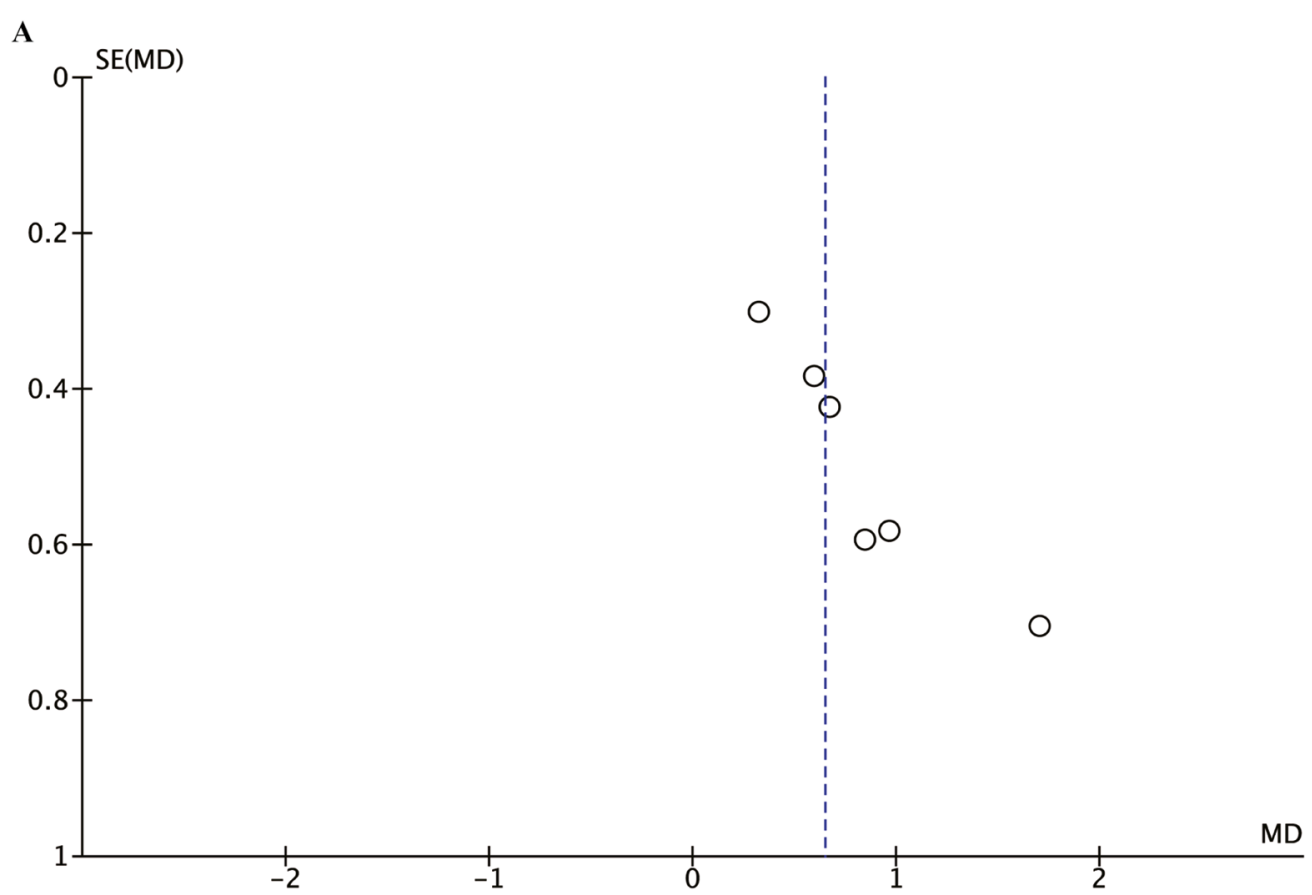

B

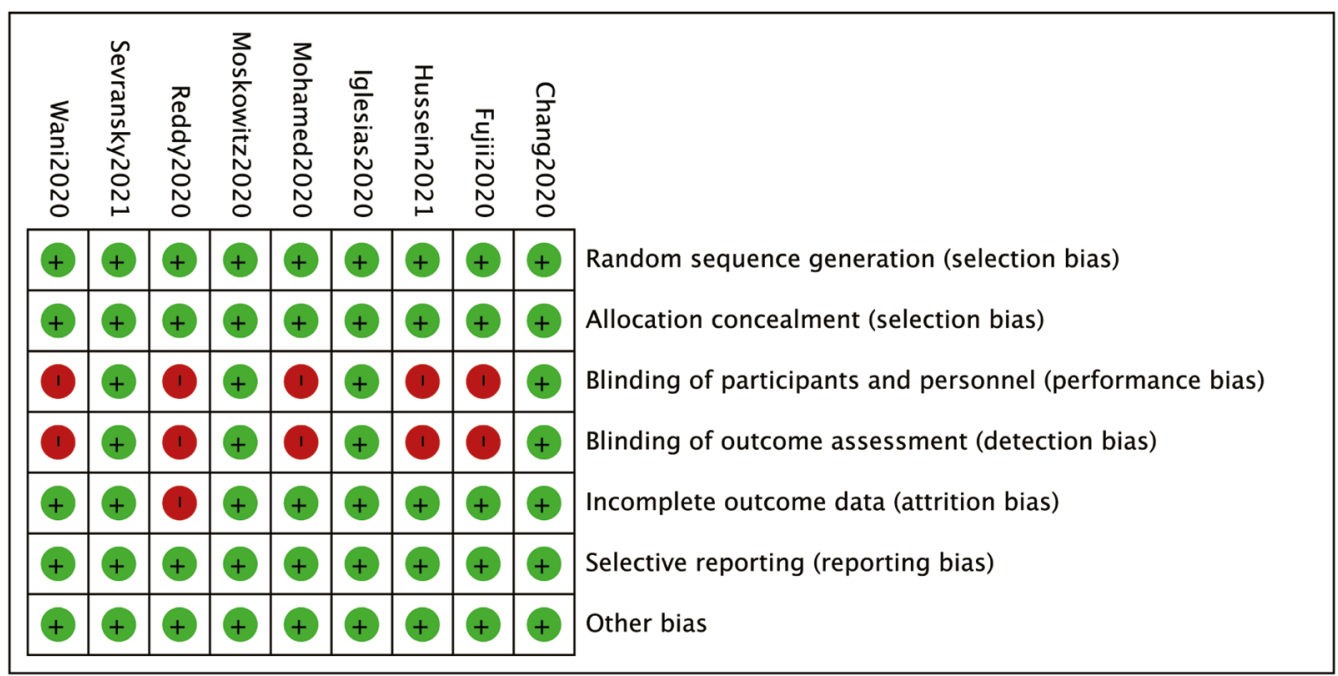

Fig. 5 Funnel plot and risk of bias summary. Legends: a Funnel plot assessing publication bias. The dots represent individual studies. b risk of bias summary for the included studies

as the primary outcome. This is the first meta-analysis that placed importance on the effects of HAT therapy on organ function. We also discussed the pathophysiologic basis and the synergistic effects for these three drugs. Moreover, we conducted TSA, subgroup analysis and sensitivity analysis to enhance methodological quality. The VICTAS trial was a large multicenter RCT, published by Sevransky et al. [21] on JAMA, which enrolled 501 patients from 43 hospital in America. With inclusion of the VICTAS trial, the results of TSA showed that the RIS was reached, and the statistical results were significant and stable. In addition, this meta-analysis is the largest at present, with nine RCTs included.

Several limitations should also be considered. First, five trials of all included were lack of blinding, which is the association with underestimation of adverse effects. Moreover, this review did not focus on side effects. The HYVCTTSSS trial was terminated at interim analysis due 
to the significant incidence of hypernatremia in the HAT group [12]. Intravenous high dose of vitamin $C$ in patients with renal failure was likely to increase oxalate, which eventually metabolized through kidneys, put their kidneys under stress [32]. Nevertheless, those could be managed in ICU. Finally, this review did not conduct more subgroup analysis. Optimal dosing time, dosage, and the administration of glucocorticoid should be considered to guide clinical practice.

\section{Conclusions}

The HAT combination improves the SOFA score in the first $72 \mathrm{~h}$ and reduces the duration of vasopressors in patients with sepsis. Given the minor mean difference of the change in the SOFA score, the mortality benefit has not been observed.

\section{Abbreviations}

HAT: Hydrocortisone, ascorbic acid, and thiamine; RCTs: Randomized controlled trials; LOS: Length of stay; TSA: Trial sequential analysis; RR: Risk ratios; MD: Mean difference; Cl: Confidence intervals; CENTRAL: Cochrane Central Register of Controlled Trials; SOFA: Sequential Organ Failure Assessment; PCT: Procalcitonin; RIS: Required information size.

\section{Supplementary Information}

The online version contains supplementary material available at https://doi. org/10.1186/s40560-021-00589-x.

Additional file 1: Appendix 1. Search strategies

\section{Acknowledgements}

None.

\section{Authors' contributions}

QD and NWL designed the study. NWL and LYC identified the studies included in the meta-analysis. NWL and SHL analyzed the data. NWL drafted the manuscript and QD revised the manuscript. All authors reviewed and approved the final manuscript.

\section{Funding}

The Special Fund of the Pediatric Medical Coordinated Development Center of Beijing Hospitals Authority. NO.XTCX201820.

\section{Availability of data and materials}

The data and materials for this meta-analysis are included in the list of references.

\section{Declarations}

Ethics approval and consent to participate

Not applicable.

Consent for publication

Not applicable.

\section{Competing interests}

We declare that there are no competing interests.

\section{Author details}

${ }^{1}$ Department of Critical Medicine, Children's Hospital Affiliated to the Capital Institute of Pediatrics, NO.2 Ya Bao Road, Chaoyang District, Beijing 100020,

China. ${ }^{2}$ Pediatric Intensive Care Unit, Children's Hospital of Fudan University, National Children's Medical Center, Shanghai 100020, China.

Received: 8 September 2021 Accepted: 1 December 2021

Published online: 18 December 2021

References

1. Singer M, Deutschman CS, Seymour CW, Shankar-Hari M, Annane D, Baue $M$, et al. The third international consensus definitions for sepsis and septic shock (Sepsis-3). JAMA. 2016;315(8):801-10.

2. Rudd KE, Kissoon N, Limmathurotsakul D, Bory S, Mutahunga B, Seymour CW, et al. The global burden of sepsis: barriers and potential solutions. Crit Care. 2018:22(1):232

3. Marik PE, Khangoora V, Rivera R, Hooper MH, Catravas J. Hydrocortisone, vitamin C, and thiamine for the treatment of severe sepsis and septic shock: a retrospective before-after study. Chest. 2017;151(6):1229-38.

4. Keh D, Boehnke T, Weber-Cartens S, Schulz C, Ahlers O, Bercker S, et al. Immunologic and hemodynamic effects of "low-dose" hydrocortisone in septic shock: a double-blind, randomized, placebo-controlled, crossover study. Am J Respir Crit Care Med. 2003;167(4):512-20.

5. Schorah CJ, Downing C, Piripitsi A, Gallivan L, Al-Hazaa AH, Sanderson MJ, et al. Total vitamin C, ascorbic acid, and dehydroascorbic acid concentrations in plasma of critically ill patients. Am J Clin Nutr. 1996;63(5):760-5.

6. Mallat J, Lemyze M, Thevenin D. Do not forget to give thiamine to your septic shock patient! J Thorac Dis. 2016;8(6):1062-6.

7. Moskowitz A, Andersen LW, Huang DT, Berg KM, Grossestreuer AV, Marik $P E$, et al. Ascorbic acid, corticosteroids, and thiamine in sepsis: a review of the biologic rationale and the present state of clinical evaluation. Crit Care. 2018;22(1):283.

8. Barabutis N, Khangoora V, Marik PE, Catravas JD. Hydrocortisone and ascorbic acid synergistically prevent and repair lipopolysaccharide-induced pulmonary endothelial barrier dysfunction. Chest. 2017;152(5):954-62.

9. Moskowitz A, Andersen LW, Huang DT, Berg KM, Grossestreuer AV, Marik $P E$, et al. Ascorbic acid, corticosteroids, and thiamine in sepsis: a review of the biologic rationale and the present state of clinical evaluation. Crit Care. 2018;22(1):1-7.

10. Marik PE, Varon J, Surani SR. Hydrocortisone, ascorbic acid and thiamine for sepsis: is the jury out? World J Diabetes. 2020;11(4):90-4.

11. Wani SJ, Mufti SA, Jan RA, Shah SU, Qadri SM, Khan UH, et al. Combination of vitamin C, thiamine and hydrocortisone added to standard treatment in the management of sepsis: results from an open label randomised controlled clinical trial and a review of the literature. Infect Dis. 2020;52(4):271-8.

12. Chang P, Liao Y, Guan J, Guo Y, Zhao M, Hu J, et al. Combined treatment with hydrocortisone, vitamin C, and thiamine for sepsis and septic shock: a randomized controlled trial. Chest. 2020;158(1):174-82.

13. Moskowitz A, Huang DT, Hou PC, Gong J, Doshi PB, Grossestreuer AV, et al. Effect of ascorbic acid, corticosteroids, and thiamine on organ injury in septic shock: the ACTS Randomized Clinical Trial. JAMA. 2020;324(7):642-50.

14. Vail EA, Wunsch H, Pinto R, Bosch NA, Walkey AJ, Lindenauer PK, et al. Use of hydrocortisone, ascorbic acid, and thiamine in adults with septic shock. Am J Respir Crit Care Med. 2020;202(11):1531-9.

15. Group P. Preferred reporting items for systematic reviews and meta-analyses (PRISMA) http://prisma-statement.org/.

16. Higgins JPT TJ, Chandler J, Cumpston M, Li T, Page MJ, Welch VA (editors). Cochrane Handbook for Systematic Reviews of Interventions version 6.0 (updated July 2019). Cochrane. 2019.

17. Wetterslev J, Jakobsen JC, Gluud C. Trial sequential analysis in systematic reviews with meta-analysis. BMC Med Res Methodol. 2017;17(1):39. 
18. Balakrishnan M, Gandhi H, Shah K, Pandya H, Patel R, Keshwani S, et al. Hydrocortisone, vitamin $C$ and thiamine for the treatment of sepsis and septic shock following cardiac surgery. Indian J Anaesth. 2018;62(12):934-9.

19. Iglesias J, Vassallo AV, Patel VV, Sullivan JB, Cavanaugh J, Elbaga Y. Outcomes of metabolic resuscitation using ascorbic acid, thiamine, and glucocorticoids in the early treatment of sepsis: The ORANGES Trial. Chest. 2020;158(1):164-73.

20. Mohamed ZU, Prasannan P, Moni M, Edathadathil F, Prasanna P, Menon $A$, et al. Vitamin c therapy or routine care in septic shock (ViCTOR) trial: effect of intravenous vitamin C, thiamine, and hydrocortisone administration on inpatient mortality among patients with septic shock. Indian J Crit Care Med. 2020;24(8):653-61.

21. Sevransky JE, Rothman RE, Hager DN, Bernard GR, Brown SM, Buchman $\mathrm{TG}$, et al. Effect of vitamin C, thiamine, and hydrocortisone on ventilatorand vasopressor-free days in patients with sepsis- and VICTAS randomized clinical trial. JAMA. 2021;325(8):742-50.

22. Fujii T, Luethi N, Young PJ, Frei DR, Eastwood GM, French CJ, et al. Effect of vitamin C, hydrocortisone, and thiamine vs hydrocortisone alone on time alive and free of vasopressor support among patients with septic shock: the VITAMINS Randomized Clinical Trial. JAMA. 2020;323(5):423-31.

23. Hussein AA, Sabry NA, Abdalla MS, Farid SF. A prospective, randomised clinical study comparing triple therapy regimen to hydrocortisone monotherapy in reducing mortality in septic shock patients. Int I Clin Pract. 2021;75(9):e14376.

24. Reddy PR, Samavedam S, Aluru N, Yelle S, Rajyalakshmi B. Metabolic resuscitation using hydrocortisone ascorbic acid thiamine: do individual components influence reversal of shock independently? Indian J Crit Care Med. 2020:24(8):649-52.

25. Lambden S, Laterre PF, Levy MM, Francois B. The SOFA score-development, utility and challenges of accurate assessment in clinical trials. Crit Care. 2019;23(1):374.

26. Seymour CW, Liu VX, Iwashyna TJ, Brunkhorst FM, Rea TD, Scherag A, et al. Assessment of clinical criteria for sepsis: for the third international consensus definitions for sepsis and septic shock (sepsis-3). JAMA. 2016;315(8):762-74.

27. Wald EL, Sanchez-Pinto LN, Smith CM, Moran T, Badke CM, Barhight MF, et al. Hydrocortisone-ascorbic acid-thiamine use associated with lower mortality in pediatric septic shock. Am J Respir Crit Care Med. 2020;201(7):863-7.

28. Liu D, Su L, Han G, Yan P, Xie L. Prognostic value of procalcitonin in adult patients with sepsis: a systematic review and meta-analysis. PLoS ONE. 2015;10(6):e0129450.

29. Houwink AP, Rijkenberg S, Bosman RJ, van der Voort PH. The association between lactate, mean arterial pressure, central venous oxygen saturation and peripheral temperature and mortality in severe sepsis: a retrospective cohort analysis. Crit Care. 2016;20:56.

30. Zayed Y, Alzghoul BN, Banifadel M, Venigandla H, Hyde R, Sutchu S, et al. Vitamin C, thiamine, and hydrocortisone in the treatment of sepsis: a meta-analysis and trial sequential analysis of randomized controlled trials. J Intensive Care Med. 2021;22:1-7.

31. Somagutta MKR, Pormento MKL, Khan MA, Hamdan A, Hange N, Kc M, et al. The efficacy of vitamin C, thiamine, and corticosteroid therapy in adult sepsis patients: a systematic review and meta-analysis. Acute Crit Care. 2021;36(3):185-200.

32. WandzilakTR, D'Andre SD, Davis PA, Williams HE. Effect of high dose vitamin C on urinary oxalate levels. J Urol. 1994;151(4):834-7.

\section{Publisher's Note}

Springer Nature remains neutral with regard to jurisdictional claims in published maps and institutional affiliations. 